Tohoku J. exp. Med., 1967, 93, 269-277

\title{
Stochastic Model of Smoluchowski as Applied to Spontaneous Discharges of Lateral Geniculate Neurons in Rats
}

\author{
Kitsuya Iwama and Ichiji Sumitomo \\ Laboratory of Neurophysiology, Institute of Higher Nervous Activity, \\ Osaka University Medical School, Osaka
}

\begin{abstract}
Spontaneous unitary discharges were recorded from the lateral geniculate body in anesthetized rats. Measurements were made on the number of discharges in successive time intervals of unit length. With the neurons in which the stationary distribution of the discharge number was found to obey the Poisson law the joint distribution of the discharge numbers of two successive time intervals was studied. The stochastic model of Smoluchowski which fits to density fluctuation of a colloidal solution was found to be applicable to interval-tointerval fluctuation of the discharge number.
\end{abstract}

When a nerve cell is discharging spontaneously, the number of discharges in a time interval of fixed length fluctuates more or less from one interval to the next. Moreover, it is seen not infrequently that there is a significant correlation between successive discharge numbers.

In this paper an attempt will be reported to apply to the spontaneous discharges of nerve cells the stochastic model which was originally developed by Smoluchowski in colloid physics. Our experiments were made on the lateral geniculate body (LGB) of anesthetized rats, and this model was found useful to describe fluctuation of the discharge number under some restricted conditions.

\section{Stochastic Model of Smoluchowski}

In the followings Smoluchowski's theory ${ }^{1,2}$ on density fluctuation of a colloidal solution will be briefly described in a form slightly modified from his original account. ${ }^{3}$ Suppose that one observes by means of an ultramicroscope a small space of volume $v$ in a large quantity of a colloidal solution and counts the number of particles in that small space intermittently at intervals of a fixed time $\tau$. The problem is to give the conditional probability $\operatorname{Pr}\left(n_{1}, n_{2}\right)$ that $n_{2}$ particles are observed in $v$ after a time $\tau$ from the instant when there were observed $n_{1}$ particles in it. Let $p$ be the probability that each particle which was found in $v$ is again found in the same $v$ after a time $\tau$ and let $\lambda$ be the average of the number of particles which enter into $v$ during a time $\tau$. Then we obtain

Received for publication, July 3, 1967. 


$$
\operatorname{Pr}\left(n_{1}, n_{2}\right)=\sum_{\nu=0}^{n 1}\left(\begin{array}{c}
n_{1} \\
\nu
\end{array}\right) p^{\nu}(1-p)^{n_{1}-\nu} \begin{gathered}
e^{-\lambda} \lambda^{\left(n_{2}-\nu\right)} \\
\left(n_{2}-\nu\right) !
\end{gathered}
$$

where the terms in the sum should be replaced by zero if $\nu<n_{\mathbf{2}}$. (For readers' convenience a detailed account of the formula (1) is given in Appendix I). If the state of the system under observation is defined by the number of particles in it, the conditional probability ( 1 ) is regarded as the transition probability that the system is transferred from the state $n_{1}$ to the state $n_{2}$.

From the transition probability as defined above one can reach the stationary distribution of the particle number in the form of the Poisson distribution, that is,

$$
\operatorname{Pr}(n)=\frac{e^{-\mu} \mu^{n}}{n !}
$$

where

$$
\mu=\frac{\lambda}{1-p}
$$

Conversely, if one assumes that the stationary distribution of the particle number is of the Poisson type, then one can find the transition probability of the particle number in the form given by (1). ${ }^{4}$

Without entering into the detailed mechanisms of spontaneous activity and its fluctuation, we now assume that the number of discharges in successive time intervals correspond to the number of particles in successive observations in Smoluchowski's model. Thus, we assume the formula (1) as giving the probability that after $n_{1}$ discharges have been observed in one time interval $n_{2}$ discharges are observed in the next time interval. The problem we are facing is to examine whether or not such is actually supported by the experimental data.

From the formulas (1) and (2) the following relations are obtained which allow us to calculate the values of $p$ and $\lambda$ from the experimental data:

(i) The average of $n_{2}$ 's following a fixed $n_{1}$ is proved to be a linear function of $n_{1}$. We find

$$
\bar{n}_{2}=\lambda+p n_{1} .
$$

(ii) The mean square of the difference between $n_{1}$ and $n_{2}$ is given by

$$
\left(n_{1}-n_{2}\right)^{2}=2 \lambda .
$$

For the proofs of the formulas (4) and (5) the readers are referred to Chandrasekhar's article. ${ }^{4}$

(iii) The correlation coefficient between $n_{1}$ and $n_{2}$ is equal to the constant $p$, that is,

$$
\rho_{n_{1}, n_{2}}=p
$$

This relation has not been presented in the previous articles in such explicit form. A proof for this simple relation is given in Appendix II. 


\section{EXPERIMENTAL}

\section{1) Methods}

Rats weighing 200-400 g were used. They were anesthetized with urethane administered intraperitoneally and fixed to a stereotaxic apparatus. To record unitary activities of the LGB cells, glass microelectrodes filled with $3 \mathrm{M} \mathrm{KCl}$ were used. To activate the LGB cells single electric pulses were given to the optic chiasm through implanted wire electrodes of bipolar type. The units which were encountered 3-5 mm below the cortical surface and responded to chiasmatic stimulation were taken as belonging to the LGB. The unit activities were fed to a cathode follower preamplifier, then to a main amplifier and were displayed on the screen of a cathode ray oscillograph. They were photographed on film moving at a speed of $5 \mathrm{~cm} / \mathrm{sec}$. Mostly the recordings of spontaneous activity were made for 30-60 sec. The counting of the discharge numbers was done with consecutive time intervals of $200 \mathrm{msec}$.

\section{2) Results}

As stated above, the transition probability (1) leads to the stationary distribution which is of the Poisson type. We, therefore, first examined the stationary distribution of the discharge number in each unit. Among a total of 13 units recorded we found five units to which the Poisson law was applicable as a good approximation. With these data we further examined whether or not the formula (1) fits to interval-to-interval fluctuation of the discharge number. A detailed description on the analysis of the data will be given citing a typical example as follows.

(1) Table 1 shows frequencies of occurrence with which the discharge number $n_{1}$ is followed by the discharge number $n_{2}$ in a series of 257 time intervals measured in one LGB unit. It is seen that there are relatively high frequencies of occurrence for the $n_{1}-n_{2}$-combinations if the values of $n_{1}$ and $n_{2}$ are close to each other. This means that there is a correlation between two discharge numbers which appear in succession.

(2) This series of the discharge numbers has the average $\mu$ at 2.50 with the variance of 2.57. Its stationary distribution is shown graphically in Fig. 1, left, and is compared with the Poisson distribution calculated with the average of 2.50 . The $\chi^{2}$-test shows that under ideal conditions some 75 per cent of comparable observations should show a worse agreement.

(3) To determine the values of $\rho$ and $\lambda$ following the formula (4), $\bar{n}_{2}$ was calculated for each $n_{1}$. In Fig. 1, right, $\bar{n}_{2}$ 's are plotted on the ordinates against $n_{1}$ 's as abscissae with a line drawn by visual inspection so as to fit to the plotted points. The line has the expression

$$
\bar{n}_{2}=1.10+0.56 n_{1} .
$$

Referring to the formula (4) we obtain 
TABLE 1. Frequencies of joint occurrence of discharge numbers $n_{1}$ and $n_{\mathrm{a}}$

\begin{tabular}{|c|c|c|c|c|c|c|c|c|c|c|}
\hline$n_{1}$ & $n_{2}$ & 0 & 1 & 2 & 3 & 4 & 5 & 6 & 7 & Sum \\
\hline & 0 & $\begin{array}{c}4 \\
(8)\end{array}$ & $\begin{array}{l}10 \\
(9)\end{array}$ & $\begin{array}{c}6 \\
(5)\end{array}$ & $\begin{array}{c}4 \\
(2)\end{array}$ & $\begin{array}{c}1 \\
(1)\end{array}$ & & & & 25 \\
\hline & . & $\begin{array}{c}12 \\
(8)\end{array}$ & $\begin{array}{c}15 \\
(17)\end{array}$ & $\begin{array}{c}11 \\
(\mathbf{I} 4)\end{array}$ & $\begin{array}{c}5 \\
(7)\end{array}$ & $\begin{array}{c}5 \\
(3)\end{array}$ & $\begin{array}{c}1 \\
(1)\end{array}$ & 1 & & 50 \\
\hline & 2 & $\begin{array}{c}6 \\
(4)\end{array}$ & $\begin{array}{c}12 \\
(15)\end{array}$ & $\begin{array}{c}25 \\
(21)\end{array}$ & $\begin{array}{c}11 \\
(15)\end{array}$ & $\begin{array}{c}8 \\
(7)\end{array}$ & $\begin{array}{c}2 \\
(2)\end{array}$ & $\begin{array}{c}1 \\
(1)\end{array}$ & & 65 \\
\hline & 3 & $\begin{array}{c}3 \\
(1)\end{array}$ & $\begin{array}{c}6 \\
(7)\end{array}$ & $\begin{array}{c}13 \\
(13)\end{array}$ & $\begin{array}{c}12 \\
(13)\end{array}$ & $\begin{array}{c}9 \\
(9)\end{array}$ & $\begin{array}{c}2 \\
(3)\end{array}$ & $\begin{array}{c}1 \\
(1)\end{array}$ & 1 & 47 \\
\hline & $\vdots$ & (1) & $\begin{array}{c}5 \\
(3)\end{array}$ & $\begin{array}{c}7 \\
(8)\end{array}$ & $\begin{array}{c}9 \\
\text { (11) }\end{array}$ & $\begin{array}{c}9 \\
(9)\end{array}$ & $\begin{array}{c}5 \\
(8)\end{array}$ & $\begin{array}{c}1 \\
(2)\end{array}$ & $\begin{array}{c}1 \\
(1)\end{array}$ & 40 \\
\hline & 5 & & $\begin{array}{c}2 \\
(1)\end{array}$ & $\begin{array}{c}2 \\
(2)\end{array}$ & $\begin{array}{c}6 \\
(4)\end{array}$ & $\begin{array}{c}4 \\
(5)\end{array}$ & $\begin{array}{c}1 \\
(3)\end{array}$ & $\begin{array}{c}2 \\
(2)\end{array}$ & $\begin{array}{c}1 \\
(1)\end{array}$ & 18 \\
\hline & i & & & (1) & $\begin{array}{c}1 \\
\text { (1) }\end{array}$ & $\begin{array}{c}3 \\
(2)\end{array}$ & $\begin{array}{c}3 \\
(2)\end{array}$ & $\underset{(1)}{1}$ & (1) & 8 \\
\hline & . & & & & & $\begin{array}{c}1 \\
(1)\end{array}$ & $\begin{array}{c}1 \\
(1)\end{array}$ & $\underset{(1)}{1}$ & & 3 \\
\hline
\end{tabular}

The discharge number was measured with the time interval of 200 msec. The theoretical frequencies of occurrence (parenthesized figures) are given by counting fractions of 0.5 and over as a whole and cutting the rests.
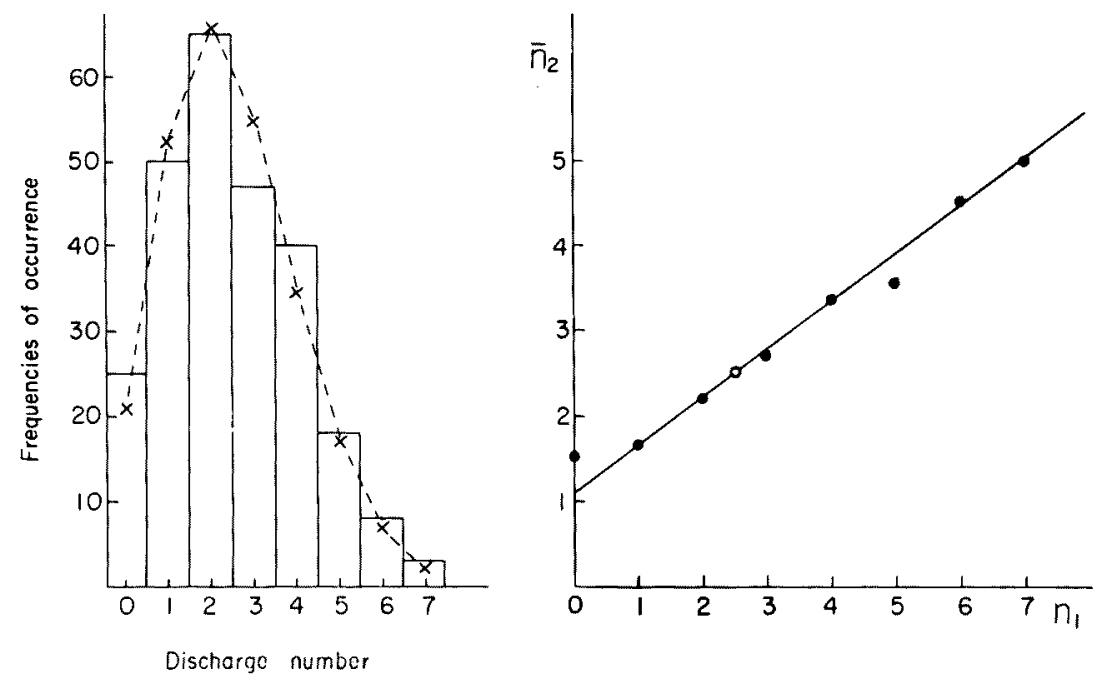

Fig. 1. Left, distribution of the discharge numbers in 257 time intervals of 200 msec length. The materials are the same as in Table 1. Average, 2.50. Variance 2.57. The Poisson distribution with the average 2.50 is shown by a broken line. Right, average of the discharge numbers $\left(\tilde{n}_{2}\right)$ following a fixed $n_{1}$ as a function of $n_{1}$. The materials are the same as in Table 1 . The plotted points are fitted by a line, $\bar{n}_{2}=1.10+0.56 n_{1}$. This line is drawn so as to pass the point with the coordinates $\bar{n}_{2}=n_{1}=\mathbf{2 . 5 0}$. 


$$
p=0.56 \text { and } \lambda=1.10 \text {. }
$$

By rearranging the formula (3) the relation $\mu=\lambda+\mu p$ is obtained. Referring to the formula (4) we know that the discharge numbers following the discharge number of $\mu$ is expected to be equal to $\mu$ on the average. The line connecting the points of $\bar{n}_{2}$ 's must pass the point $n_{1}=n_{2}=\mu(=2.50)$.

(4) Another set of the values of $p$ and $\lambda$ were obtained by calculating $\rho_{n_{1}}, n_{2}$ and $\left(\overline{n_{1}-n_{2}}\right)^{2}$. They are

$$
p=0.50 \text { and } \lambda=1.21 \text {. }
$$

These values are very close to those determined in Fig. 1, right, by the graphical method.

(5) The transition probability of the discharge number was calculated from the formula ( 1 ) by inserting $p=0.56$ and $\lambda=1.10$. The expected frequencies of occurrence for each combination of $n_{1}$ and $n_{2}$ are shown in Table 1 with parenthesized figures. It is seen that the calculated frequencies of occurrence are good approximations to the observed ones.

(6) The average life time of the state characterized by the discharge number of $n$ is given by the equation

$$
T_{n}=\frac{\tau}{1-\operatorname{Pr}(n, n)}
$$

In this formula $\operatorname{Pr}(n, n)$ is given by inserting $n_{1}=n_{2}=n$ into the general formula of transition probability (1). If an experimentally observed series of the discharge numbers is 'shuffled', it is turned into an artificial series which has no correlation between successive discharge numbers. In such series the average life time of the state is expected to follow the formula

$$
T_{n}=\stackrel{\stackrel{\tau}{\operatorname{Pr}(n)}}{1-}
$$

$\operatorname{Pr}(n)$ of which is given by the formula (2). In table 2 the observed average life times are given for different $n$ 's (second column) with the calculated ones by the formula (7) (third column) and by the formula (8) (fourth column). The theoretical values of the formula (7) are good approximations to the observed ones except for some $n$ 's. In contrast, the values obtained by the formula (8) are generally smaller than the observed ones. This is because they are obtained by disregarding the fact that in an actually obtained series of the discharge numbers the same, or about the same, discharge numbers tend to appear in succession.

(7) The average recurrence time of the state $n$ is given by

$$
\theta_{n}=T_{n} \frac{1-\operatorname{Pr}(n)}{\operatorname{Pr}(n)}
$$

where $T_{n}$ is given by the formula (7) and $\operatorname{Pr}(n)$ is by the formula (2). The observed 
TABLE 2. Average life times of the states with different discharge numbers

\begin{tabular}{c|c|c|c}
\hline Discharge number & Observed & Calculated by (7) & Calculated by (8) \\
\hline 0 & 1.19 & 1.50 & 1.09 \\
1 & 1.45 & 1.53 & 1.26 \\
2 & 1.62 & 1.48 & 1.34 \\
3 & 1.34 & 1.39 & 1.27 \\
4 & 1.29 & 1.30 & 1.15 \\
5 & 1.06 & 1.24 & 1.07 \\
6 & 1.14 & 1.16 & 1.03 \\
7 & 1.00 & 1.12 & 1.01
\end{tabular}

Average life times are given in multiples of $200 \mathrm{msec}$. The materials are the same as those of Table 1 .

TABLE 3. Average recurrence times of the states with different discharge numbers

\begin{tabular}{ccc}
\hline Discharge number & Observed & Calculated \\
0 & 16.3 & 16.7 \\
1 & 5.8 & 5.9 \\
2 & 4.9 & 4.3 \\
3 & 5.9 & 5.2 \\
4 & 5.4 & 8.5 \\
5 & 11.5 & 16.1 \\
6 & 24.8 & 40.5 \\
7 & 48.0 & 110.9
\end{tabular}

Average recurrence times are given in multiples of $200 \mathrm{msec}$. The materials are the same as those of Table 1.

and theoretical $\theta_{n}$ 's are shown in the second and third columns in Table 3, respectively. The agreement between the observed and theoretical values is not good. Such is particularly true for the states with large discharge numbers. This is probably because the series of the discharge numbers under study has not a sufficiently large number of samples (The derivation of the formulas (7), (8) and (9) are given in references 4 and 8 ).

There are four other units in which the stationary distribution of the discharge number was fitted by the Poisson law with about the same degree of closeness as in the above-mentioned case. In three of them the correlation between two successive discharge numbers was found statistically significant $\left(\rho_{n_{1}}, n_{2}=0.26,0.38\right.$ and 0.43$)$. The applicability of the formula (1) was satisfactorily supported in these cases.

\section{Discussion}

In this paper we made a study on fluctuation of spontaneous activity of the rat $L G B$ neurons in terms of the discharge number. There were found some units in which the transition probability of the discharge number is expressed by Smoluchowski's model with a good fitness. That the units which support the applica- 
bility of this model are few in number is simply due to the fact that this model requires the Poisson law as the stationary distribution and this requirement is not fulfilled in many LGB neurons. Therefore, we must admitt that the model proposed here is of limited use so far as the LGB is concerned.

It has been reported on some types of the nerve cell that the time interval of the spontaneous discharge distributes following an exponential function. ${ }^{5-7}$ It is evident that in these nerve cells the discharge number in a time interval of unit length distributes following the Poisson law. It may be of some interest to test the applicability of Smoluchowski's model in these cases.

The constant $p$ in the transition probability of Smoluchowski's model is given a clear physical meaning when applied to density fluctuation of a colloidal solution; it depends upon the geometry of the space under observation, the radius of colloid particles and so on. When Fürth ${ }^{8}$ applied Smoluchowski's model to fluctuation of the number of pedestrians in a segment of a street, the constant $p$ was related to the walking speed of the pedestrian and the extent of the street segment under observation. On the other hand, though this model was found applicable to fluctuation of the number of earthquakes in successive time intervals of unit length, ${ }^{9}$ the physical meaning of the constant $p$ in this case remains unexplained. In applying Smoluchowski's model to fluctuation of spontaneous activity of nerve cells, the constant $p$ must be interpreted in terms of neuron physiology. This is not reached as yet.

\section{APPENDIX}

I. On the transition probability $\operatorname{Pr}\left(n_{1}, n_{2}\right)$

Let $n$ be the number of particles which are found in the space $v$ at one observation. Among them, $(n-x)$ particles move away from the space $v$ during a time $\tau$, leaving $x$ particles in it. Assuming that particles have the same probability $p$ to remain in the space $v$ and they behave independently, this event is given a probability

$$
\left(\begin{array}{l}
n \\
x
\end{array}\right) p^{x}(1-p)^{n-x}
$$

which is a succession of $n$ Bernoulli trials.

Besides the particles moving away, there are ones which enter into the space $v$ during a time $\tau$. The number of entrants is assumed to vary following the Poisson law with the average $\lambda$, hence the probability that $y$ particles enter into $v$ is given by

$$
\frac{e^{-\lambda} \lambda^{y}}{y !}
$$

Let us now consider that during a time $\tau$ from the instant when $n_{1}$ particles were found, $\left(n_{1}-v\right)$ particles move away and $\left(n_{2}-v\right)$ particles enter into $v$, resulting a joint occurrence of the particle numbers $n_{1}$ and $n_{2}$. The probability for this 
event is obtained by combining the above-mentioned Bernoulli and Poisson distributions. Thus we obtain

$$
\sum_{j=0}^{n_{1}}\left(\begin{array}{c}
n_{1} \\
\nu
\end{array}\right) p(1-p)^{n_{1}-\nu} \frac{e^{-\lambda} \lambda{ }^{\left(n_{2}-\nu\right)}}{\left(n_{2}-\nu\right) !},
$$

where $\nu \leqq n_{1}$ if $n_{1} \leqq n_{2}$ and $\nu \leqq n_{2}$ if $n_{1} \geqq n_{2}$.

II. On the correlation coefficient $\rho_{n_{1}}, n_{2}$

Let the averages of $n_{1}$ and $n_{2}$ be $\mu_{1}$ and $\mu_{2}$, respectively, and let the standard deviations of $n_{1}$ and $n_{2}$ be $\sigma_{1}$ and $\sigma_{2}$, respectively. The covariance $S_{n_{1}, n_{2}}$ of $n_{1}$ and $n_{2}$ is defined as follows:

$$
S_{n_{1}, n_{2}}=\left(n_{1}-\mu_{1}\right)\left(\overline{n_{2}}-\overline{\mu_{2}}\right) .
$$

Then it is evident that

$$
\begin{aligned}
S_{n_{1}, n_{2}} & =\left(n_{1}-\mu_{1}\right) n_{2} \\
& =\left(n_{1}-\mu_{1}\right)\left(\lambda+p n_{1}\right),
\end{aligned}
$$

because we have the relation (4). Finally we obtain

$$
S_{n_{1}, n_{2}}=p\left(n-\mu_{1}\right) n_{1}=p \sigma_{1}^{2} .
$$

Using the covarience $S_{n_{1}}, n_{2}$ the correlation coefficient $\rho_{n_{1}}, n_{2}$ between $n_{1}$ and $n_{2}$ is written in the form of

$$
\rho_{n_{1}, n_{2}}=\frac{S_{n_{1}, n_{2}}}{\sigma_{1} \sigma_{2}}
$$

Since $S_{n_{1}, n_{2}}=p \sigma_{1}^{2}$, we find

$$
\rho_{n_{1}, n_{2}}=p
$$

because $\sigma_{1}$ and $\sigma_{2}$ are taken as equal to each other as the number of samples are very large.

\section{Acknowledgment}

Our thanks are due to Dr. Tadashi Okamoto, Professor of Mathematical Statistics, Faculty of Basic Engineering, Osaka University, who kindly discussed the problem with us.

\section{References}

1) Smoluchowski, M.v. Ủber die zeitliche Verändlichkeit der Gruppierung von Emulsionsteilehen und Reversibilität der Diffusionserseheinungen. Physikal. Z., 1915, 16, 321327.

2) Simoluchowski, M.v. Drei Vorträge üher Diffusion, Brownsche Molekularbewegungen und Koagulation von Kolloidteilehen. Physikal. Z., 1916, 17, 557-571.

3) Feller, W. An introluction to probability theory and its application. Vol. 1, John Wiley \& Sons, Inc., New York, 1957, p. 377.

4) Chandrasekhar, S. Stochastic problems in physics and astronomy. Rev. Mod. Physics, 1943, 15, 1-89.

5) Martin, A.R. \& Branch, C.L. Spontaneous activity of Betz cells in eats with midbrain 
lesions. J. Neurophysiol., 1958, 21, 368-379.

6) Hunt, C.C. \& Kuno, M. Background discharges and evoked responses of spinal interneurones. J. Physiol. (Lond.), 1959, 147, 364-384.

7) Oomura, Y., Ooyama, H., Yamamoto, T. \& Naka, F. Reciprocal relationship of the lateral and ventromedial hypothalamus in the regulation of food intake. Physiol. \& Behav., 1967, 2, 97-115.

8) Fürth, R. Statistik und Wahrscheinlichkeitsnachwirkung. Physikal. Z., 1918, 19, $421-426$; $1919,20,21$.

9) Kishinouye, F. \& Kawasumi, H. An application of the theory of fluctuation to problems in statistical seismology. Jishin Kenkyusho Ihoo (Report of Seismological Institute of Tokyo Imperial University), 1928, 4, 75-83 (in Japanese with English abstract). 